\title{
An Evolving Conceptual Model of the Middle Trinity Aquifer, Hays County, Central Texas
}

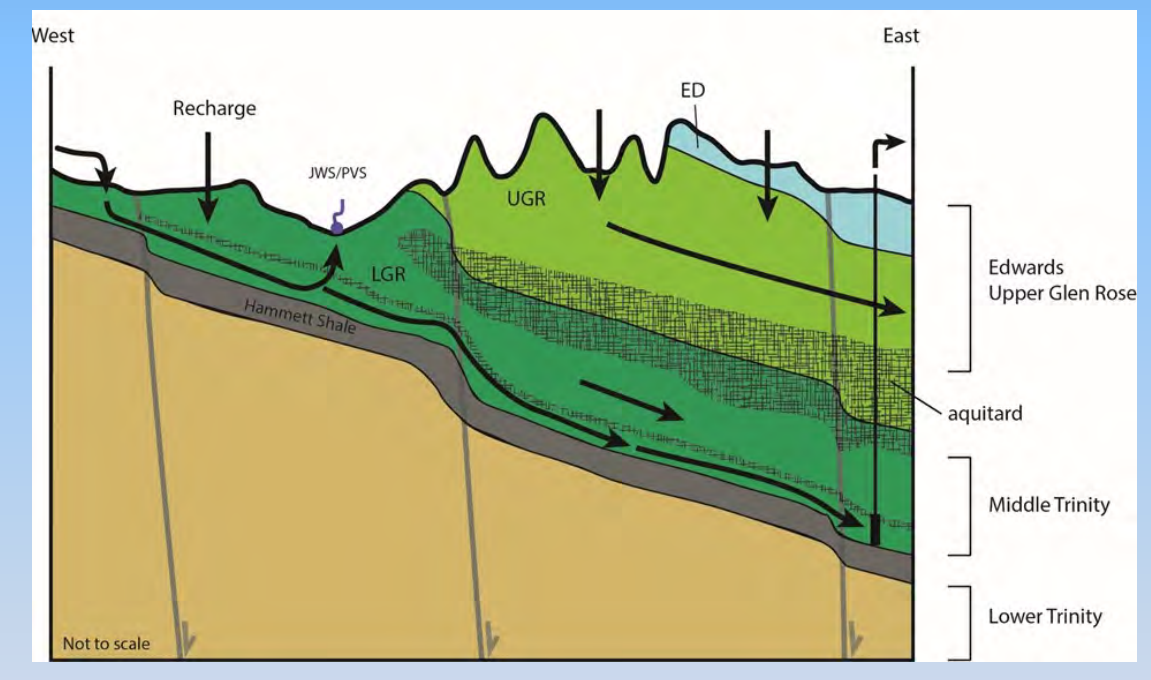

B.B. Hunt (BSEACD), B.A. Smith (BSEACD), M.O. Gary (EAA), A.S. Broun (HTGCD), D.A Wierman (TSU)

Geological Society of America South Central Meeting San Antonio, Texas March 2017 


\section{Conceptual Model}

- Description of the groundwater flow system

- Geometries

- Hydrostratigraphy

- Recharge/Discharge

- Flow paths

- Key step prior to numerical modeling,

Example: Edwards Aquifer

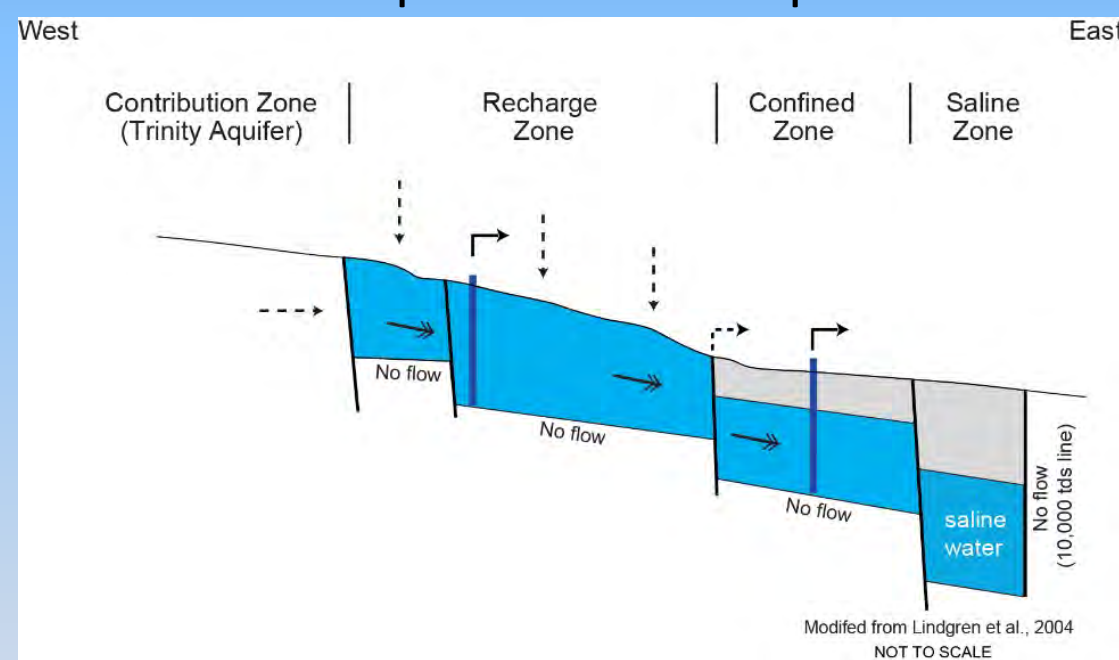
interpreting aquifer tests 


\section{Middle Trinity Aquifer}

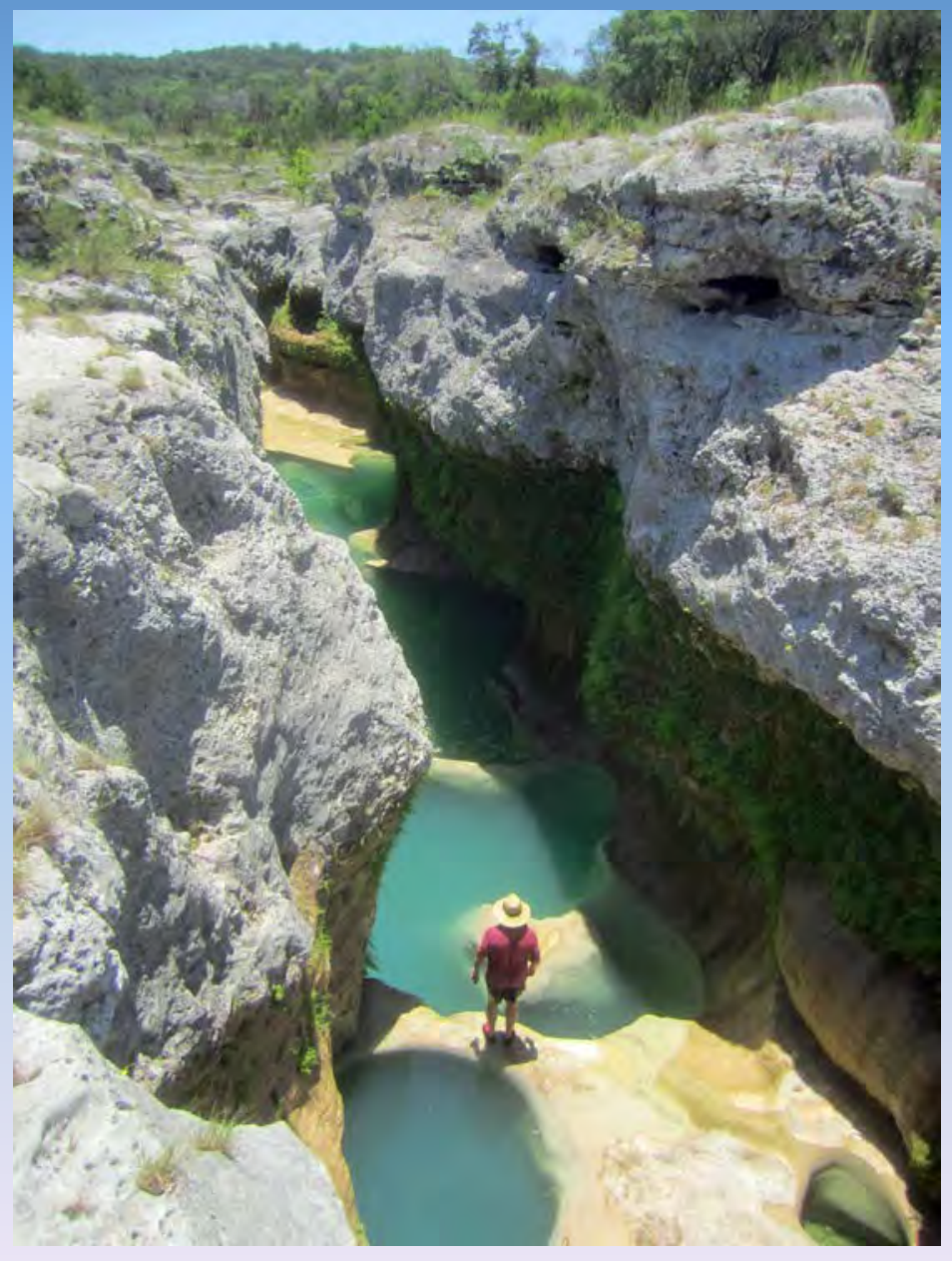

- Water supply

- Provides baseflows

- Surface water ecology

- Source of recharge to Trinity and Edwards aquifers

Lower Glen Rose, "Narrows" on Blanco River 


\section{Middle Trinity Aquifer}

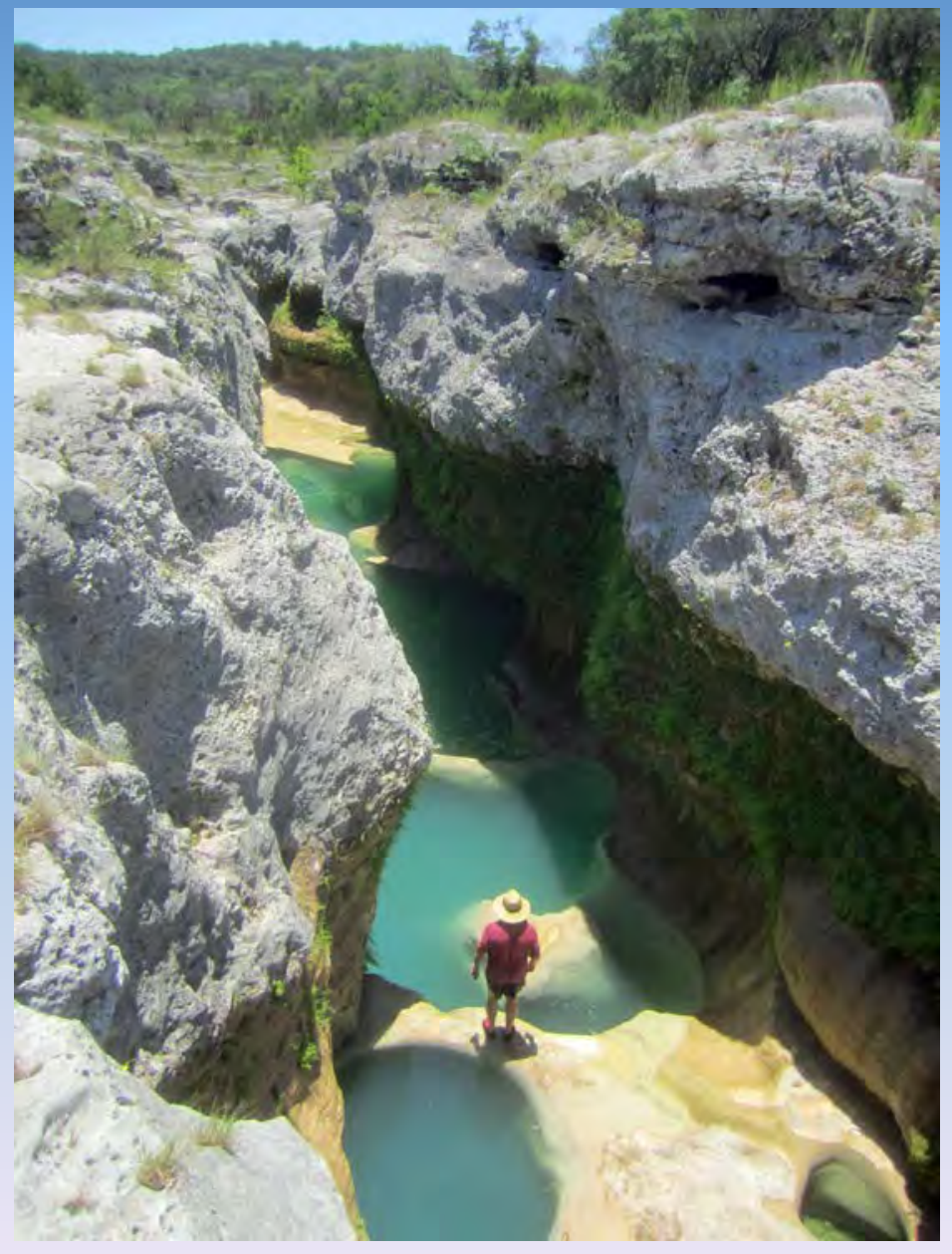

Previous conceptual and numerical models are inadequate for current needs.

Lower Glen Rose, "Narrows" on Blanco River 


\section{Trinity Aquifer}

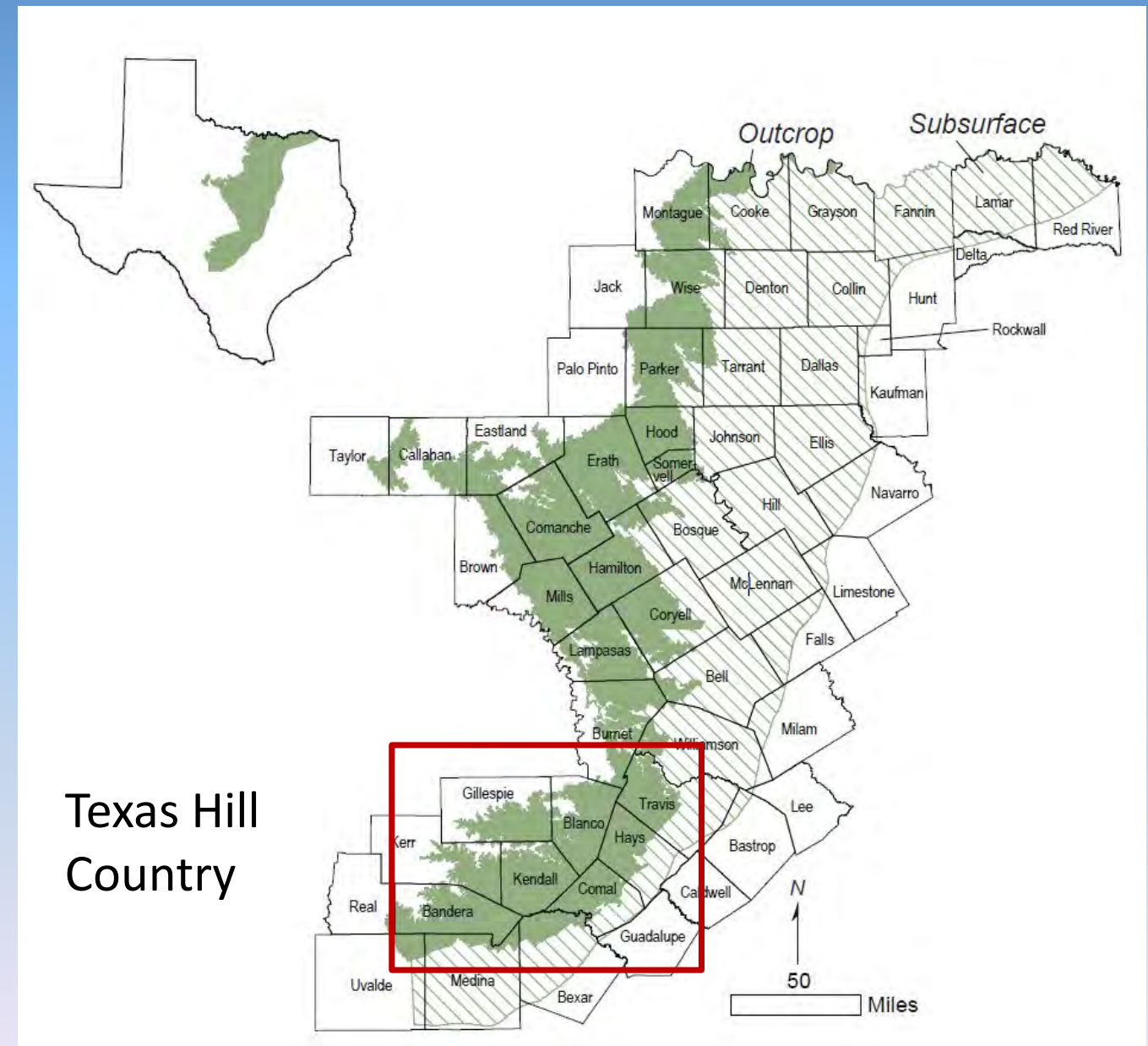

TWDB Aquifers of Texas

George et al., 2011 


\section{Major Aquifers}

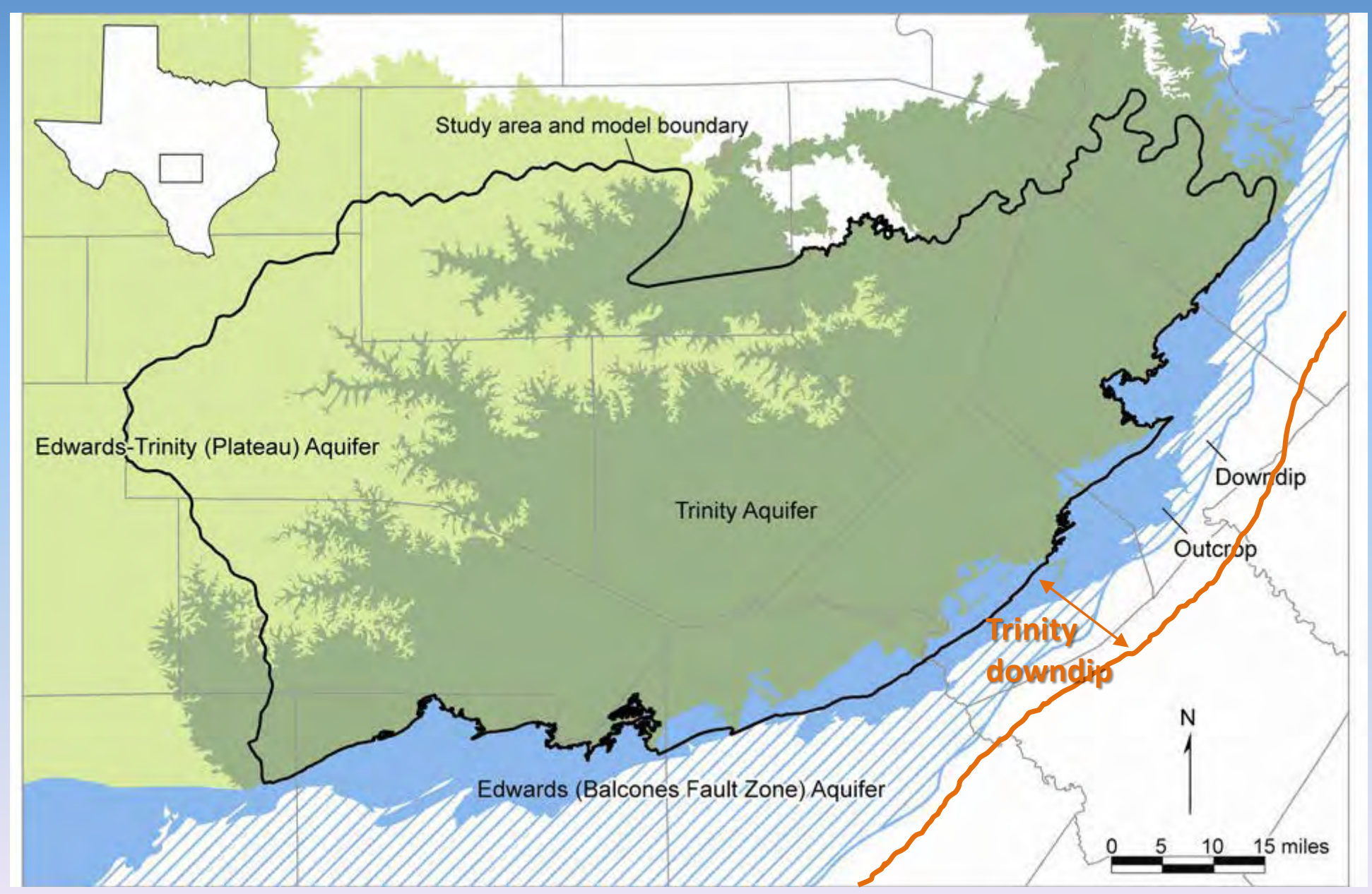

TWDB Report 377,

Jones et al., 2011, modified from Mace et al, 2000 


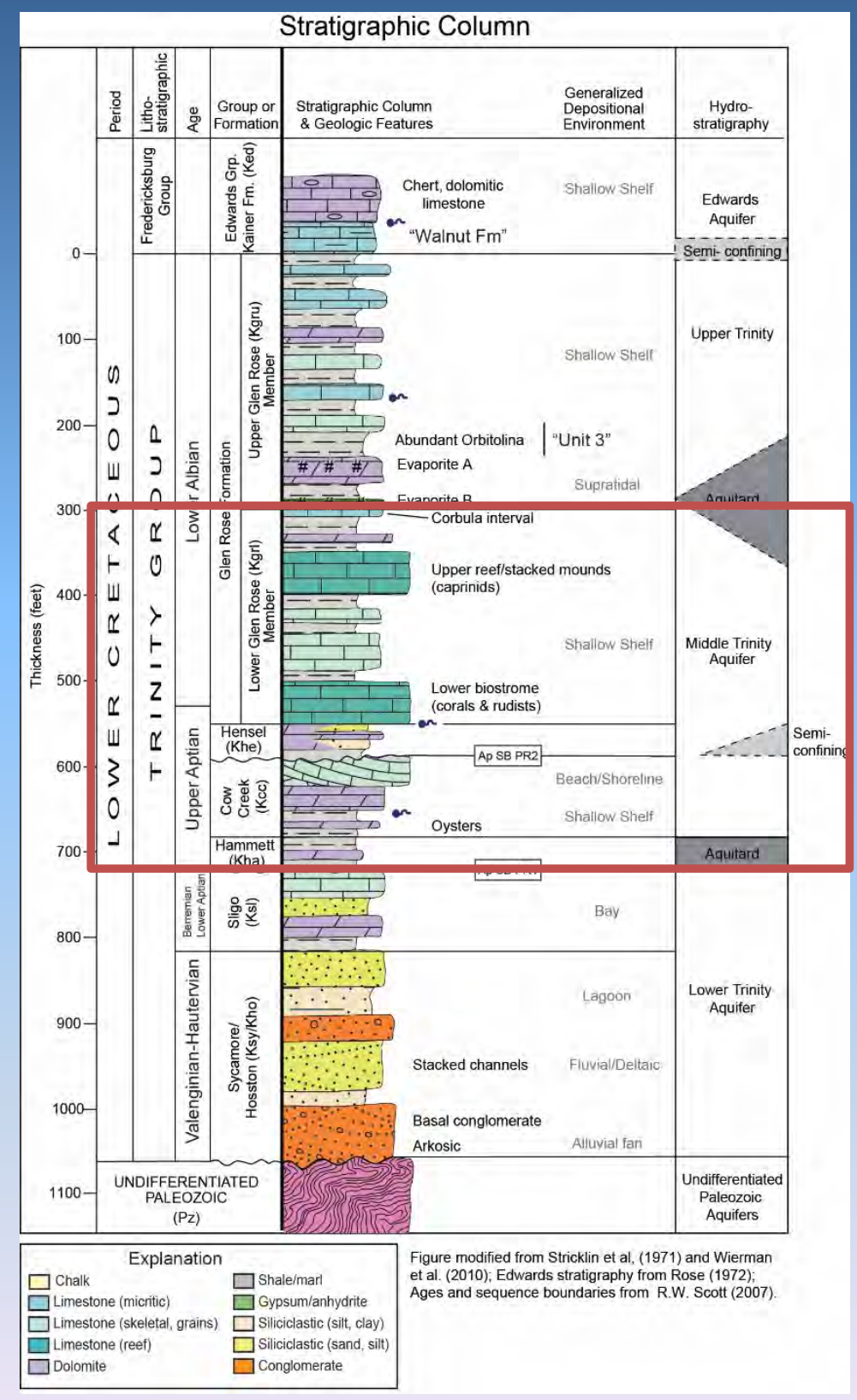

\section{Hydrostratigraphy}

Middle Trinity Aquifer

- Lower Glen Rose

- Hensel

- Cow Creek 


\section{Previous Middle Trinity Conceptual Model (Hays County)}

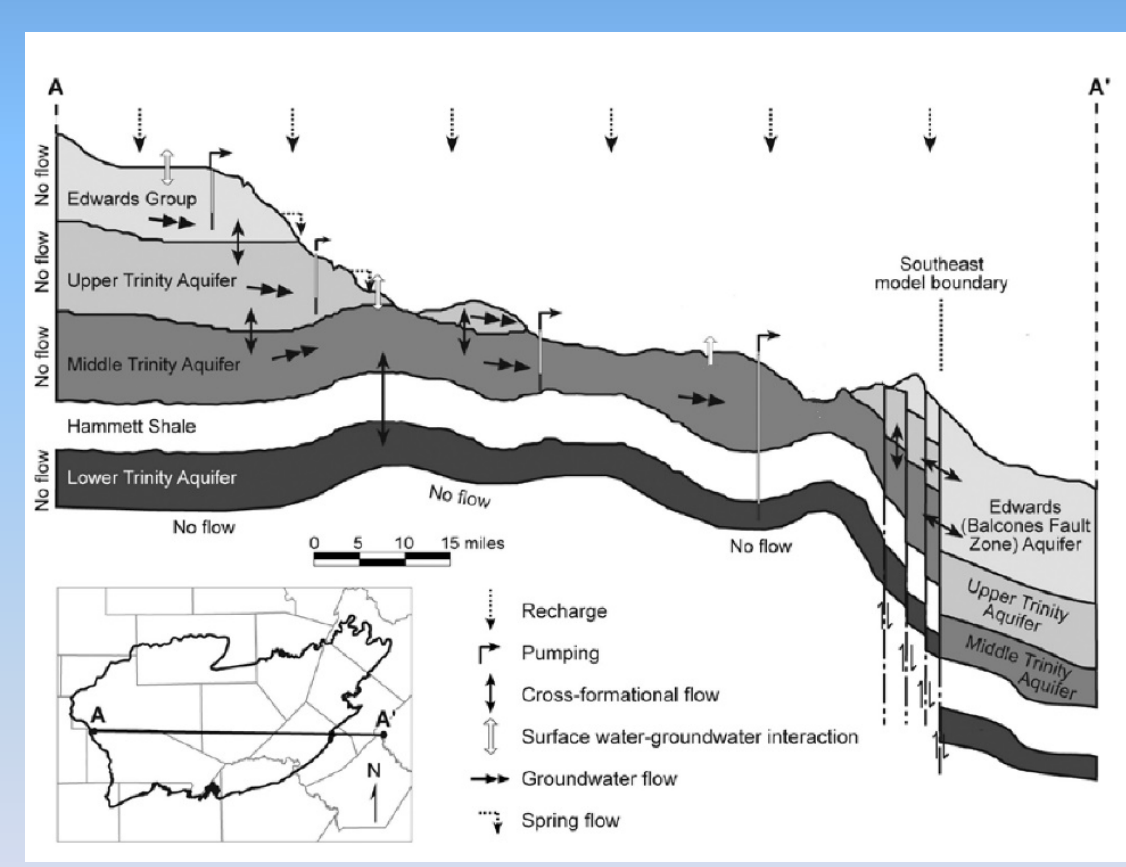

TWDB Report 377, Jones et al., 2011 modified from Mace et al., 2000.
- Diffuse recharge

- Surface and groundwater interaction limited

- Middle Trinity in BFZ not included 


\section{Working Middle Trinity Conceptual Model (Hays County)}

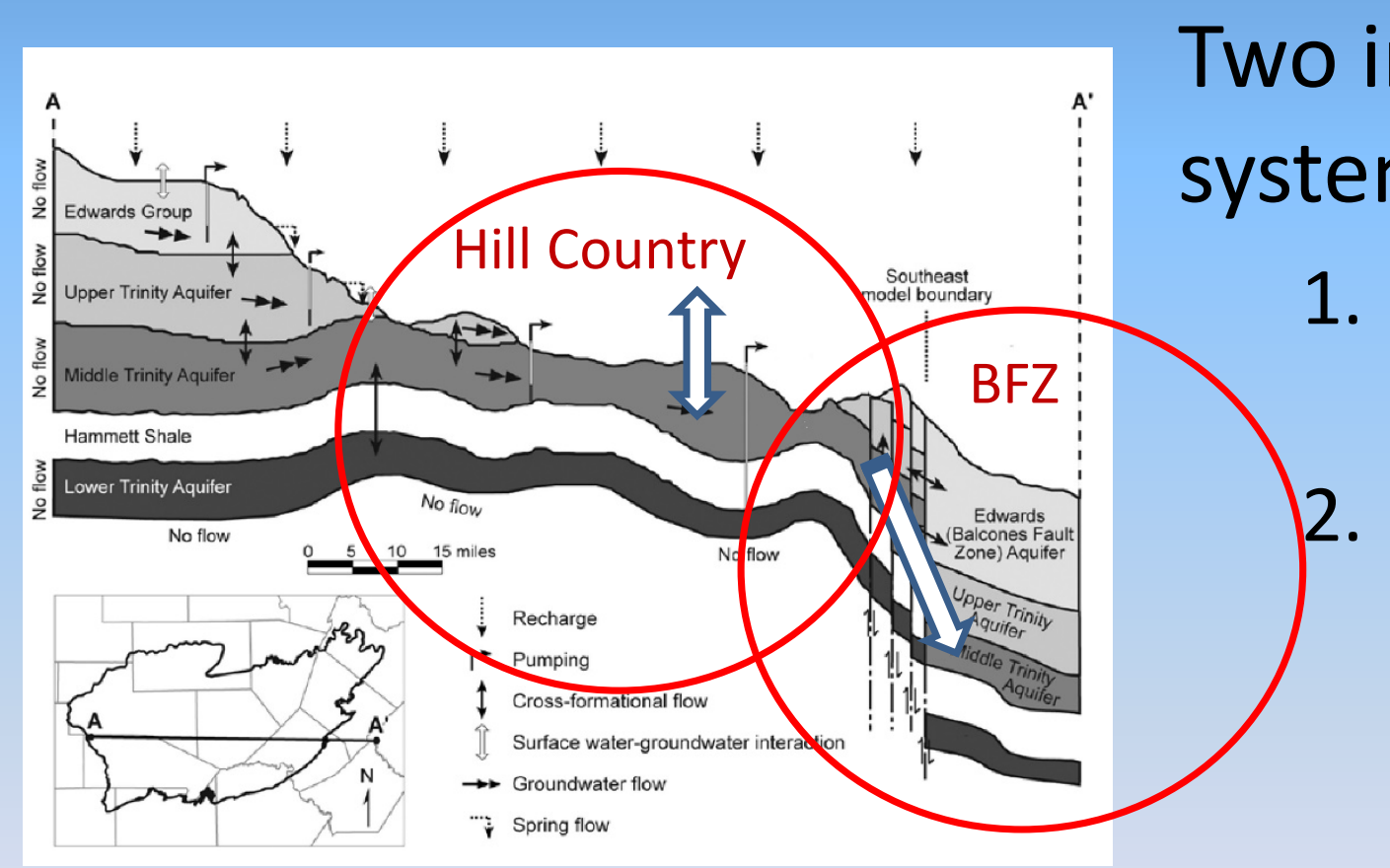

1. Hill Country Middle Trinity Aquifer

2. BFZ Middle Trinity Aquifer

TWDB Report 377, Jones et al., 2011, 


\section{Hill Country Middle Trinity Aquifer}

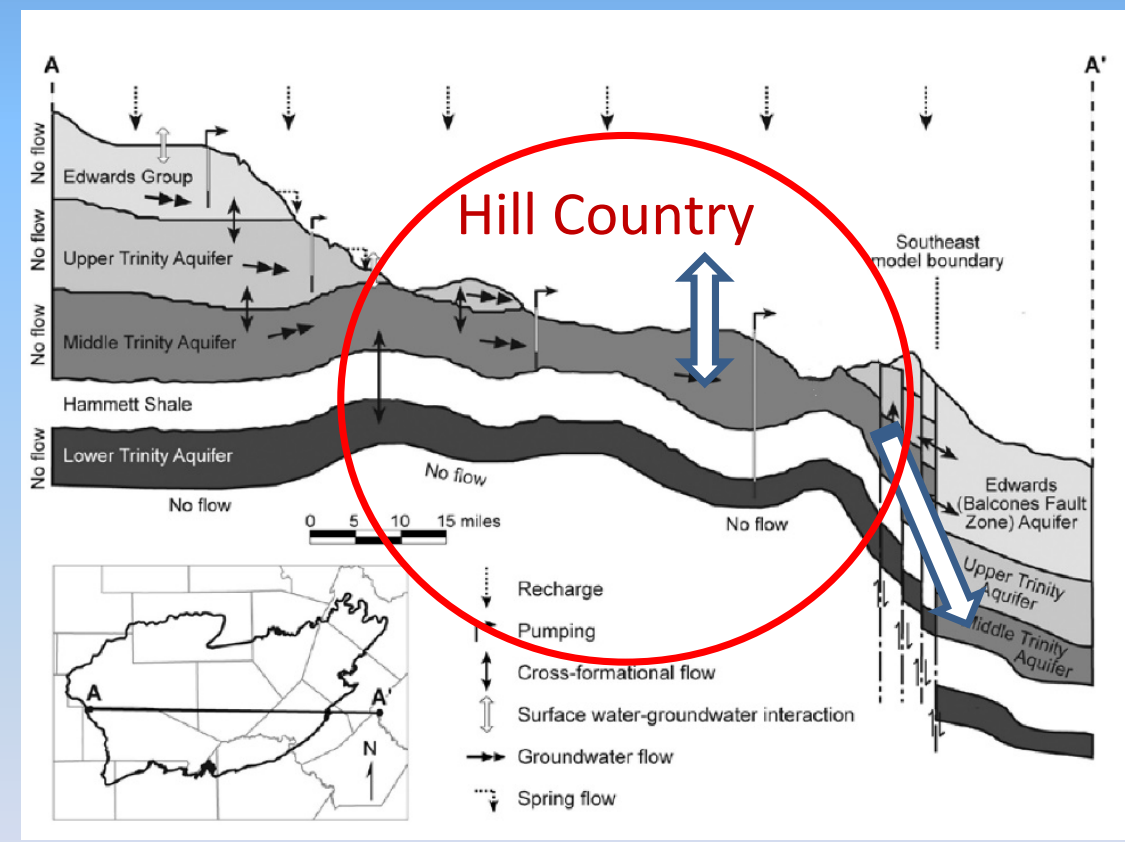

TWDB Report 377, Jones et al., 2011,
- Karstic (caves, springs)

- similar to Edwards in places

- Surface-groundwater interaction significant

- Conduit to diffuse flow

- Relatively fresh and young water 


\section{Generalized Flow Loss Map}

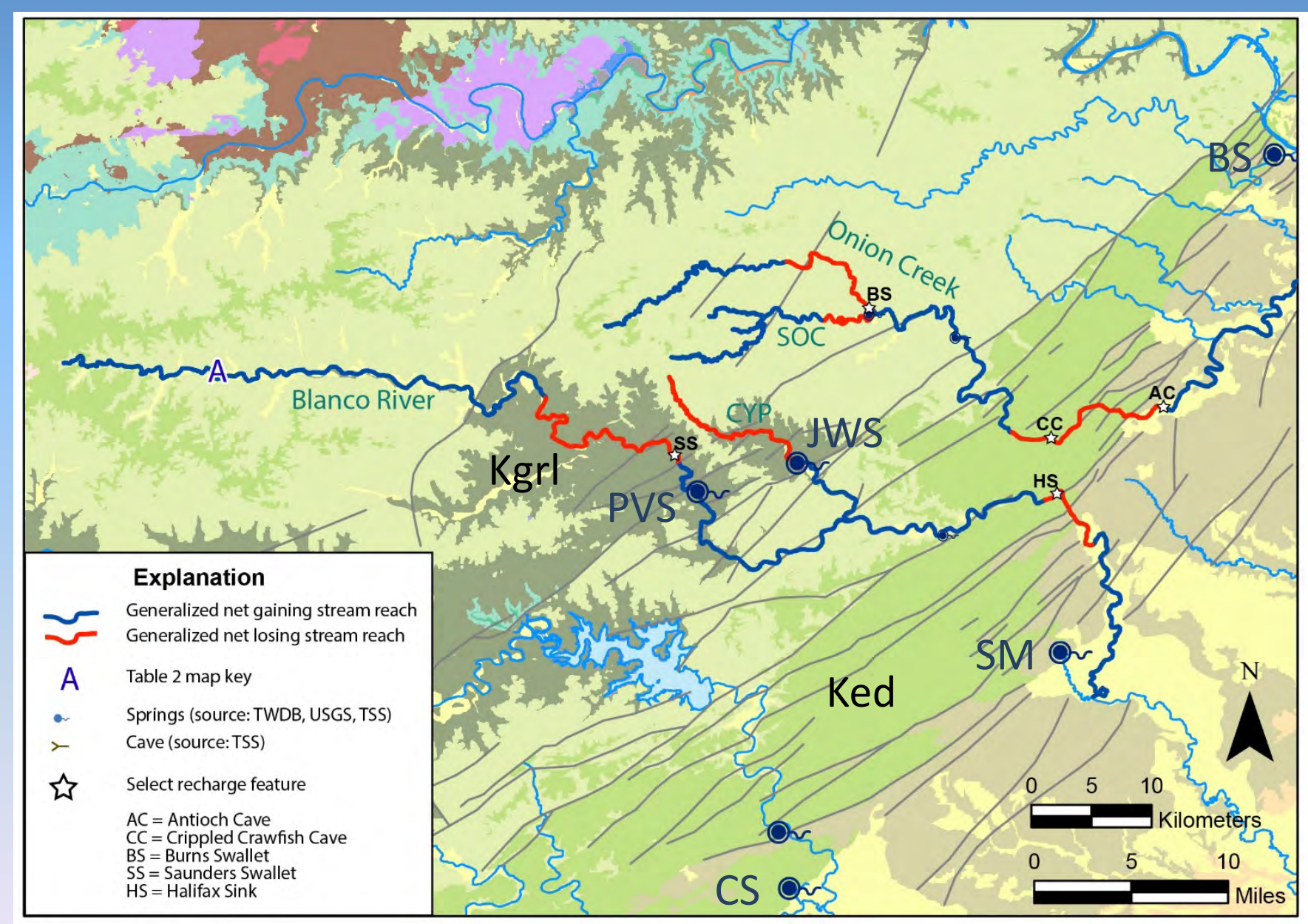




\section{Discrete Karst Recharge}

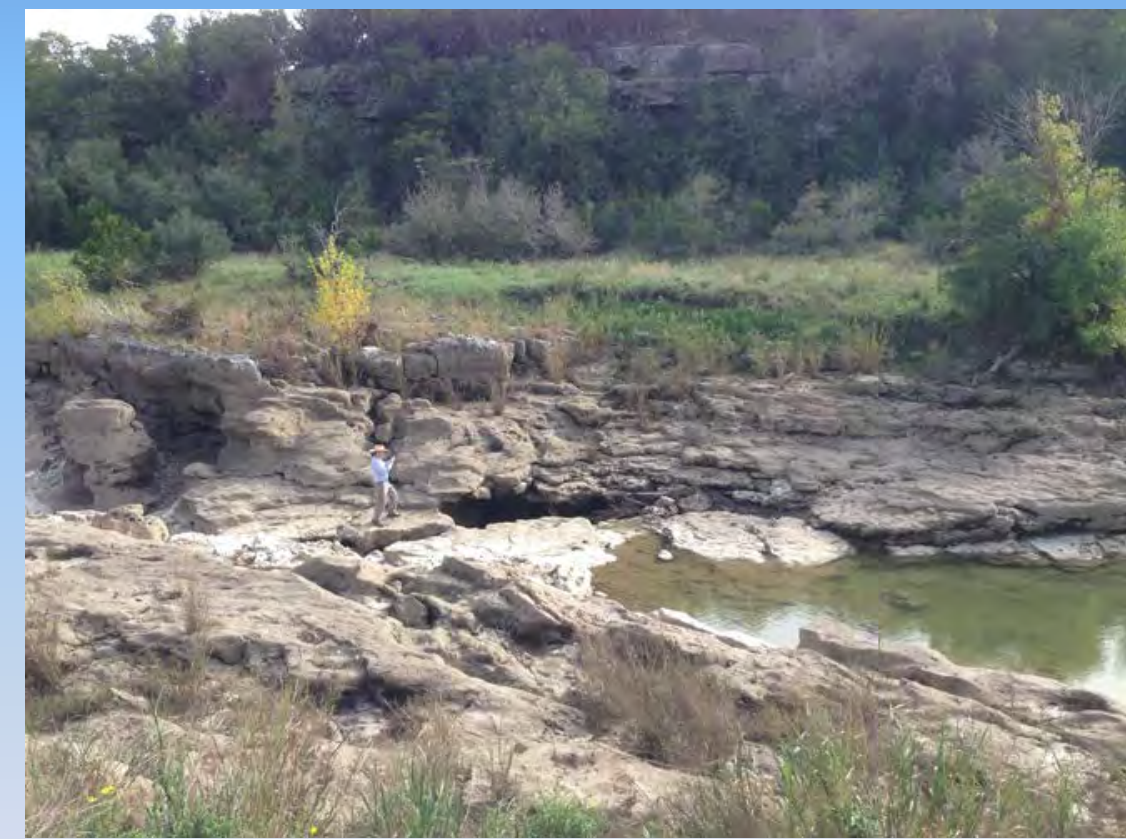

Saunder's Swallet in Cow Creek, "Dry"

Blanco River

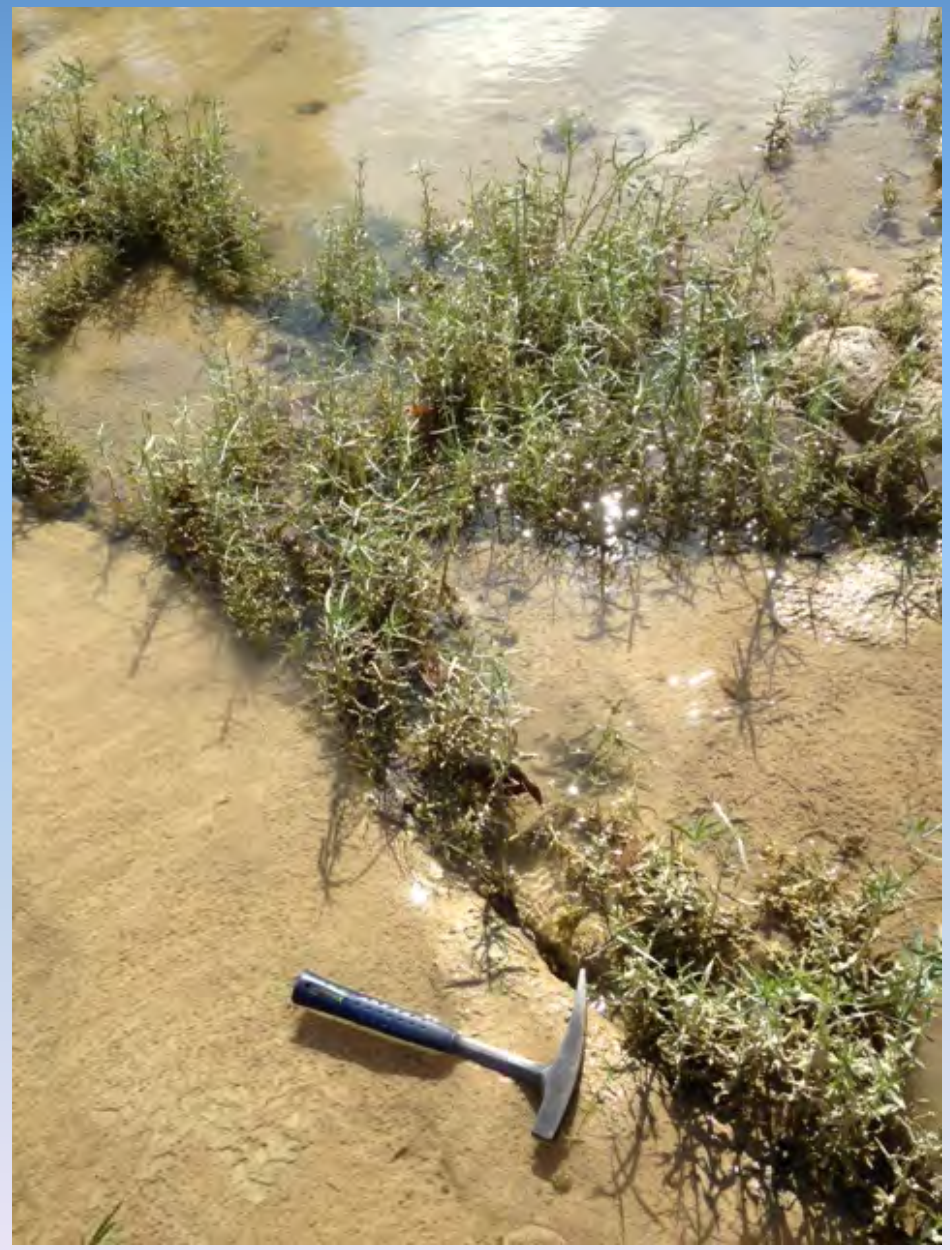

Burns Ranch Swallet, base of Upper Glen Rose, Onion Creek 


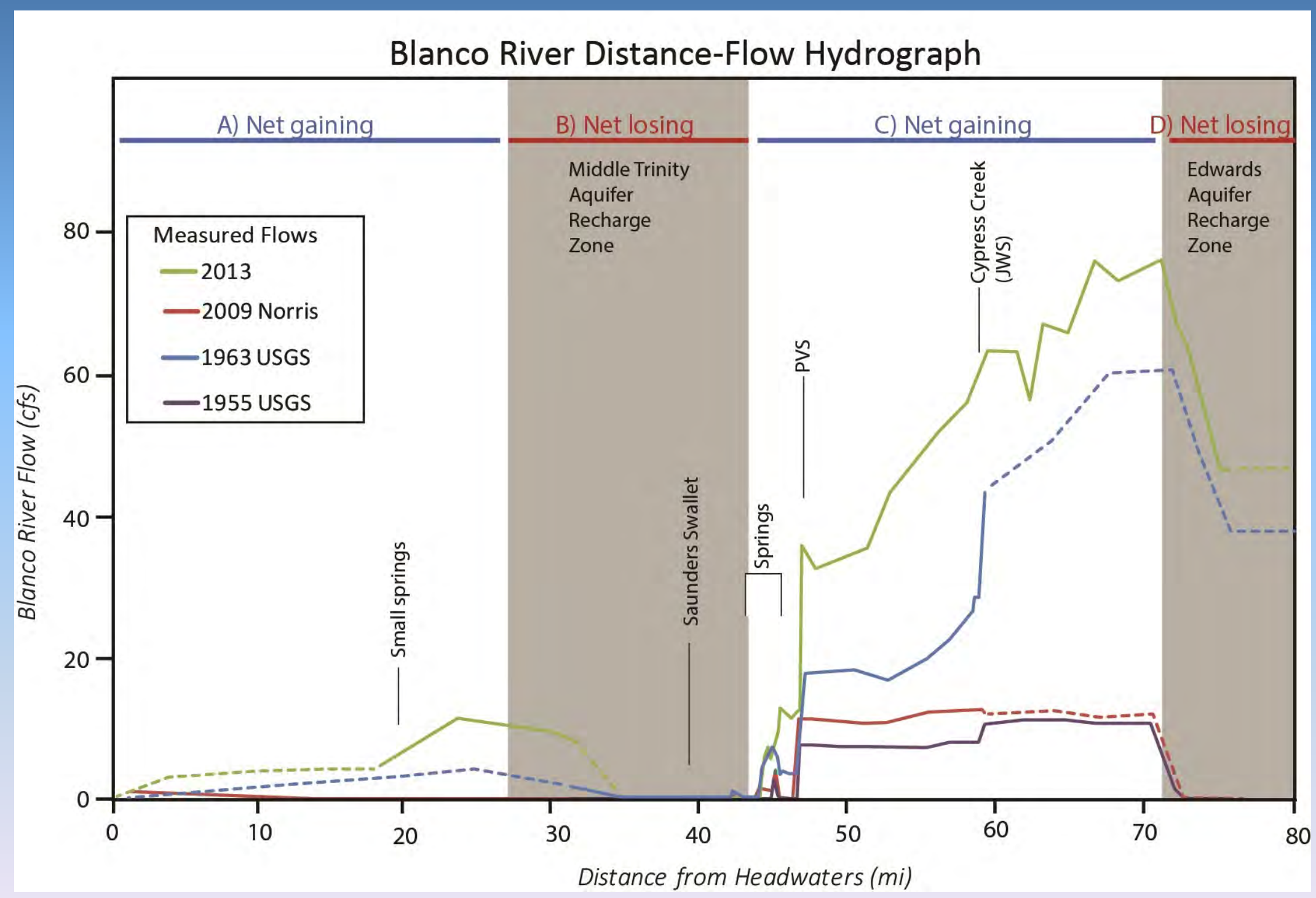




\section{Middle Trinity Groundwater Flow}

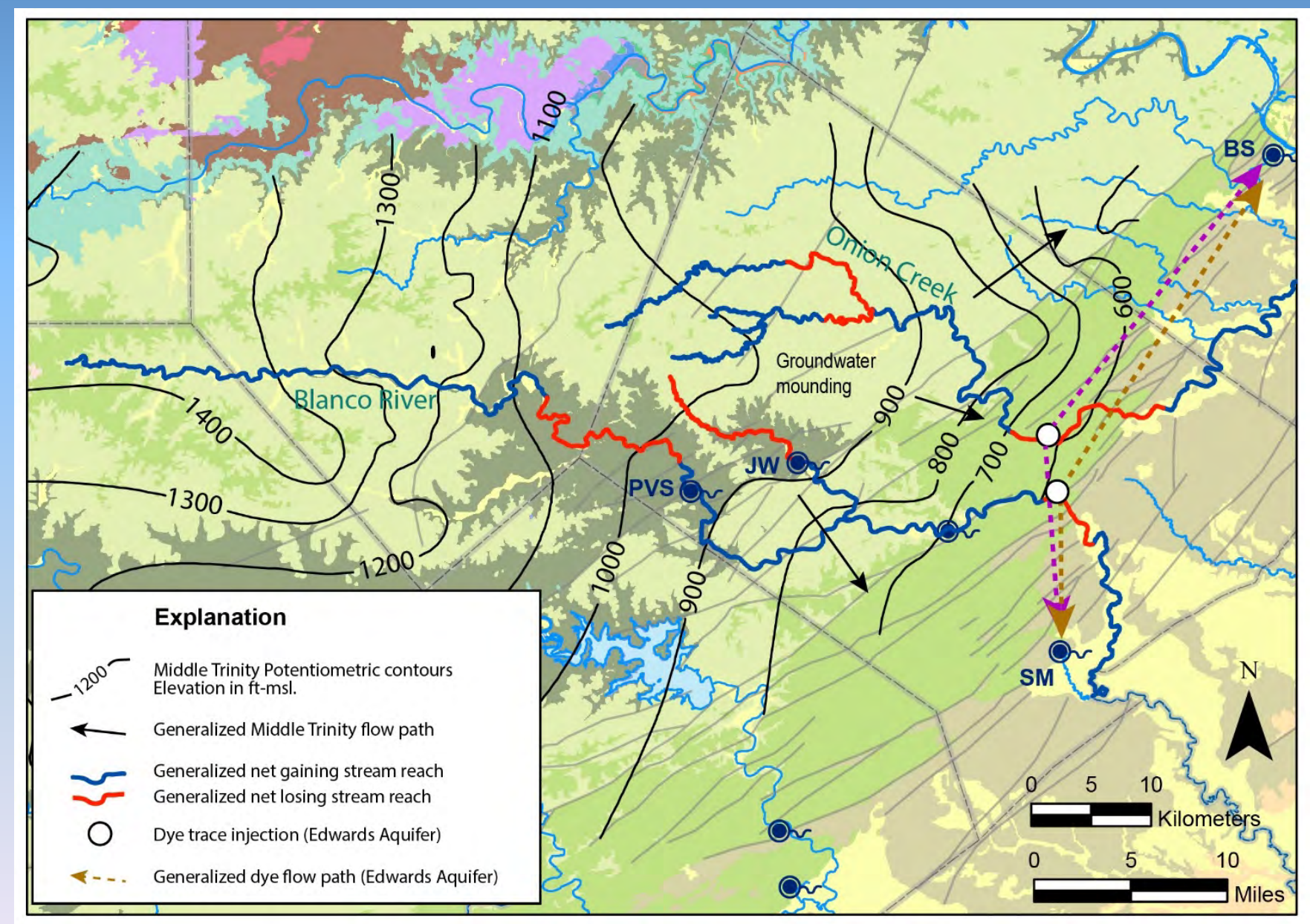




\section{Middle Trinity Groundwater Flow}

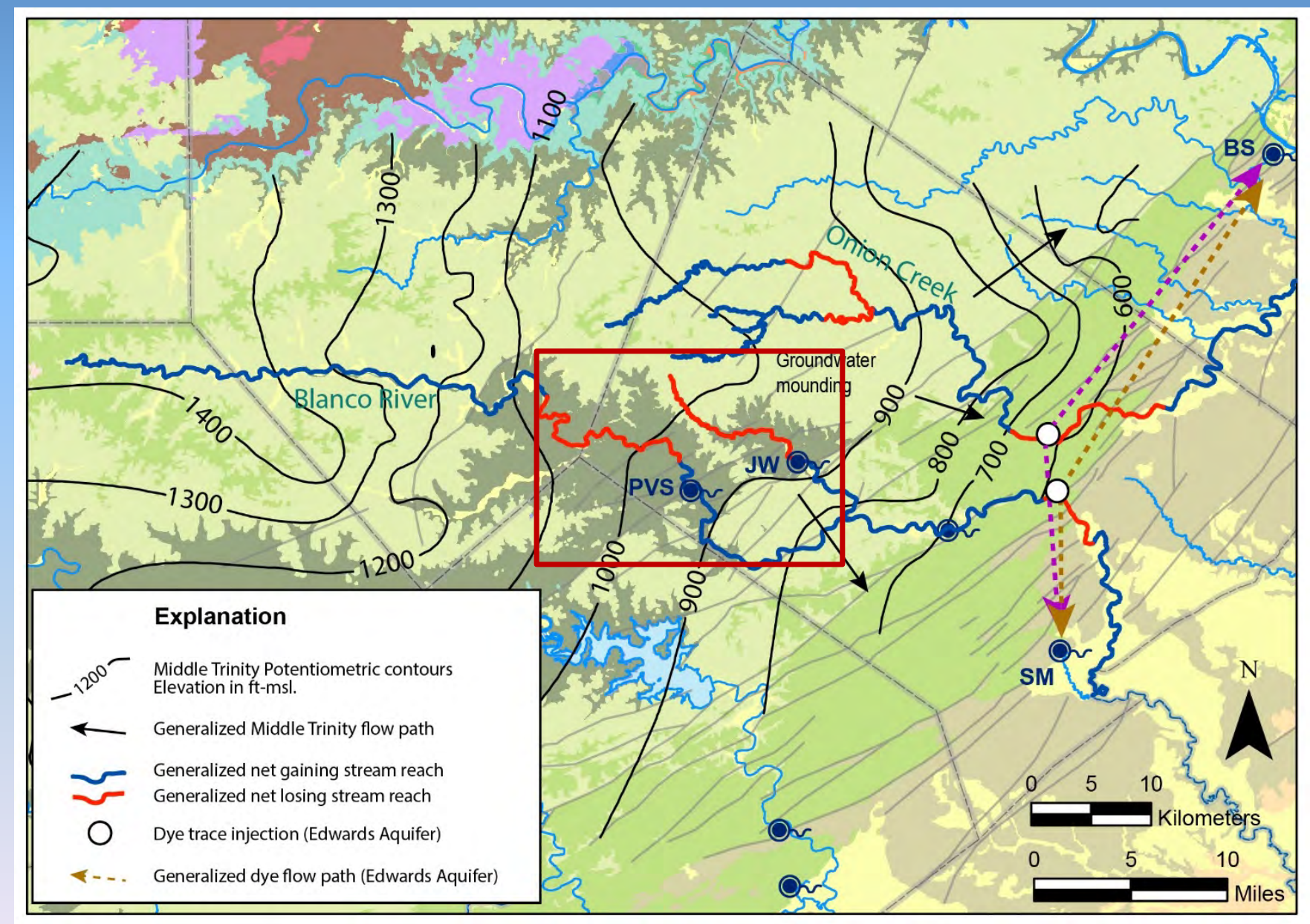




\section{Middle Trinity Groundwater Flow Blanco Valley}

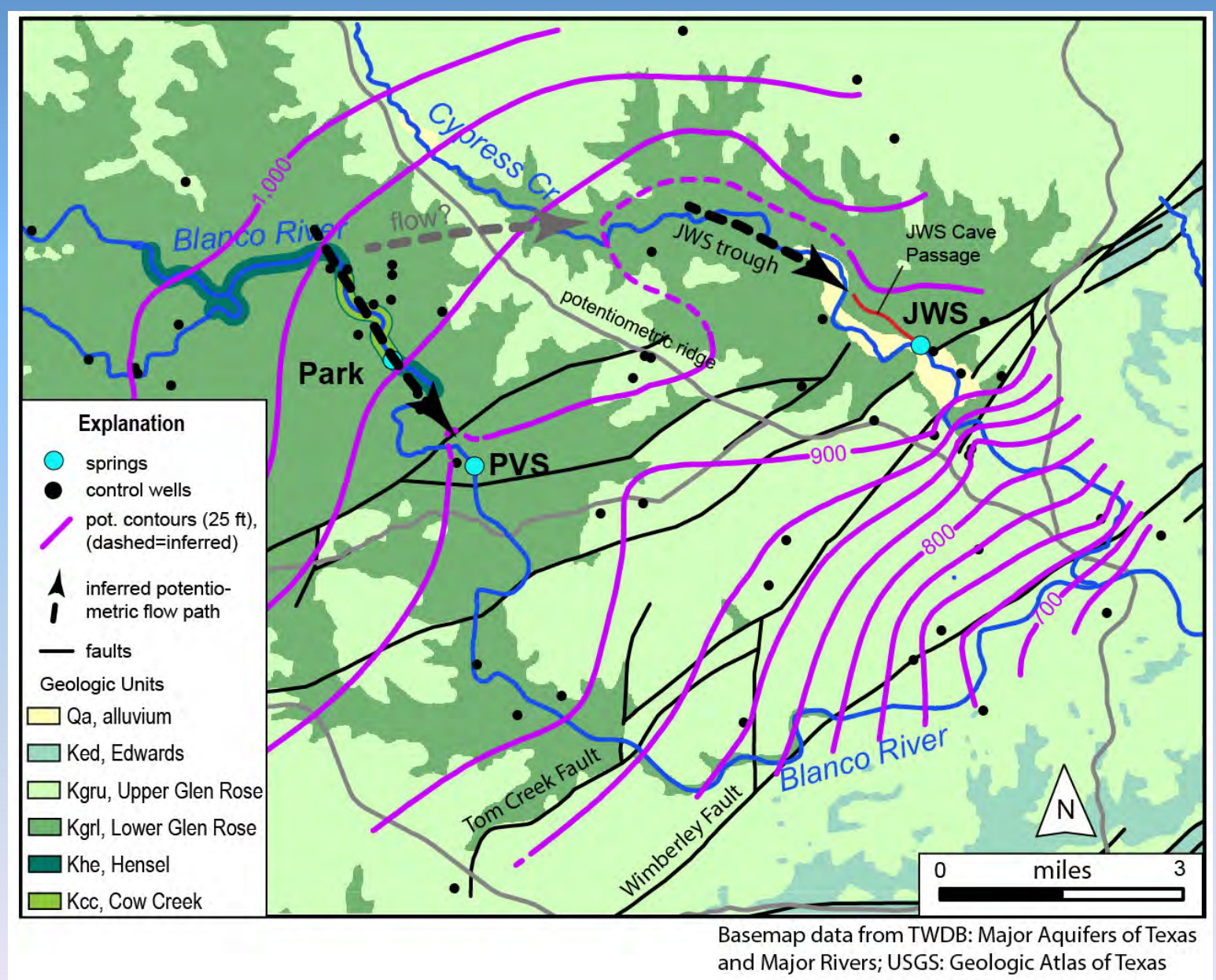

Watson et al., 2014 


\section{Springflow}

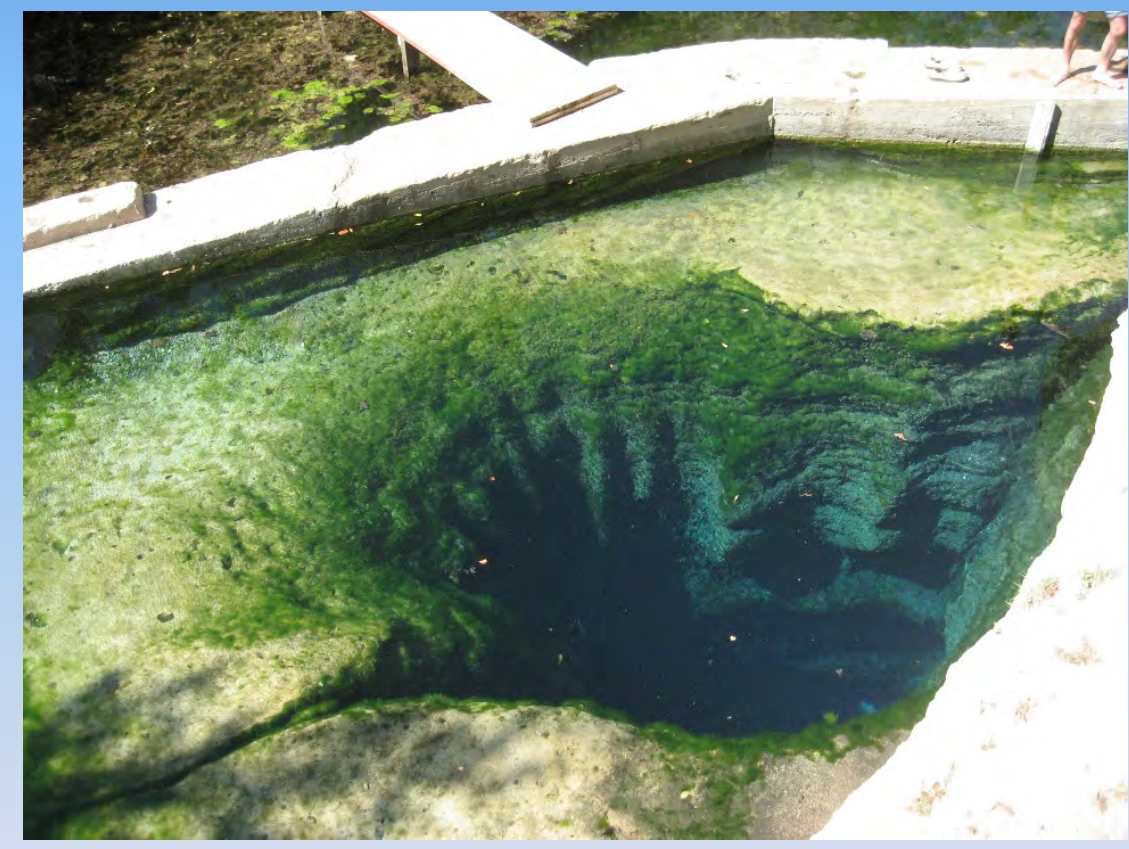

Jacob's Well Spring

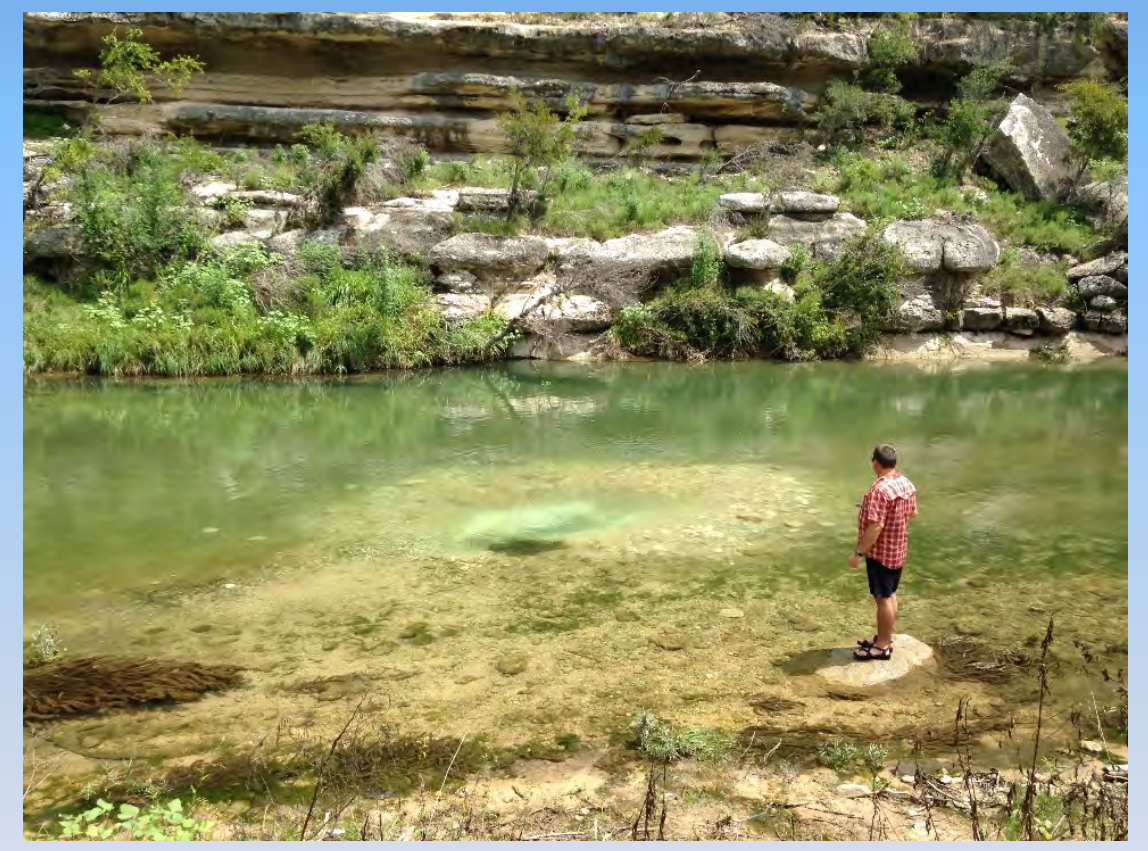

Pleasant Valley Spring 


\section{Middle Trinity TDS and Tritium Map}

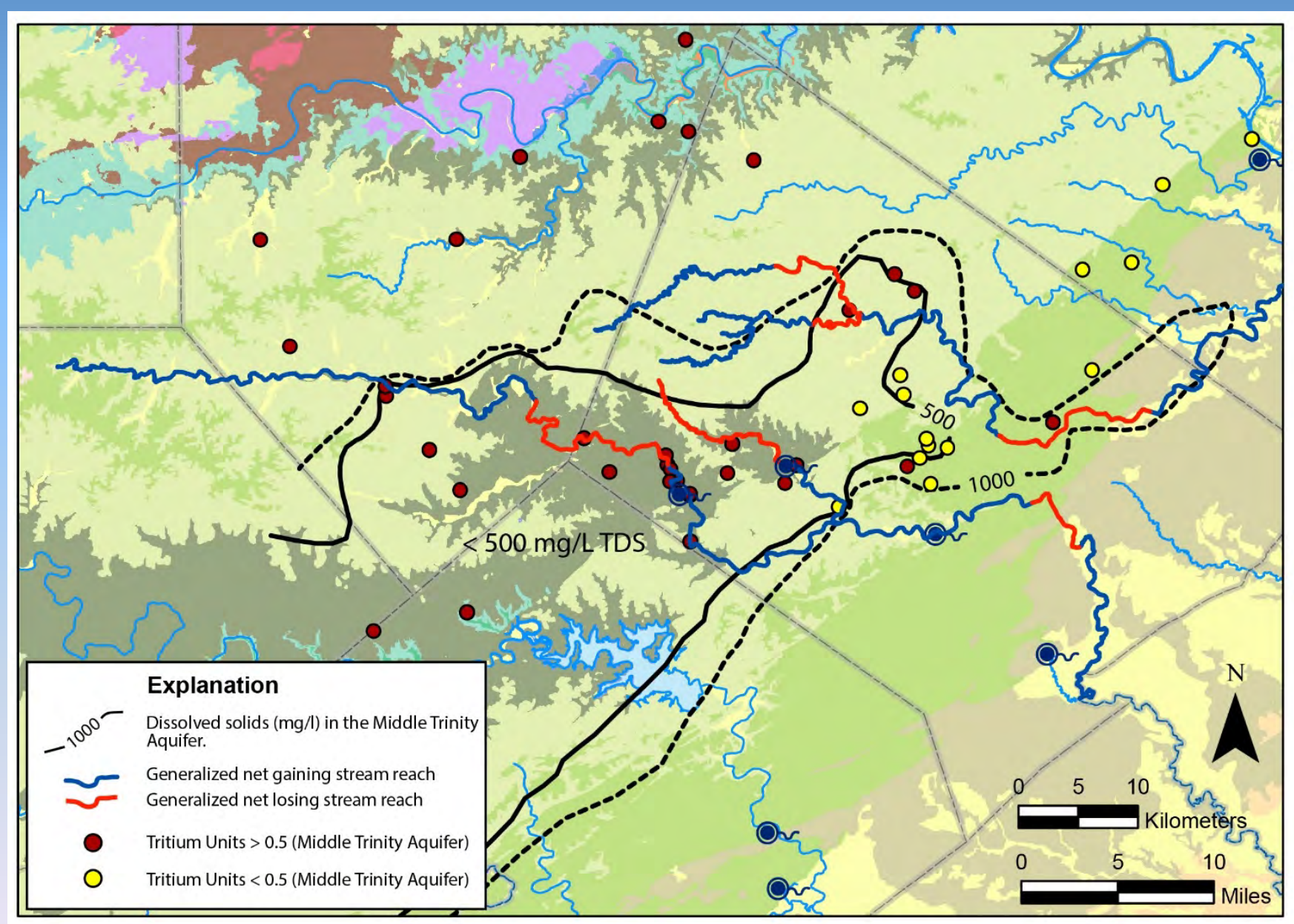




\section{BFZ Middle Trinity Aquifer}

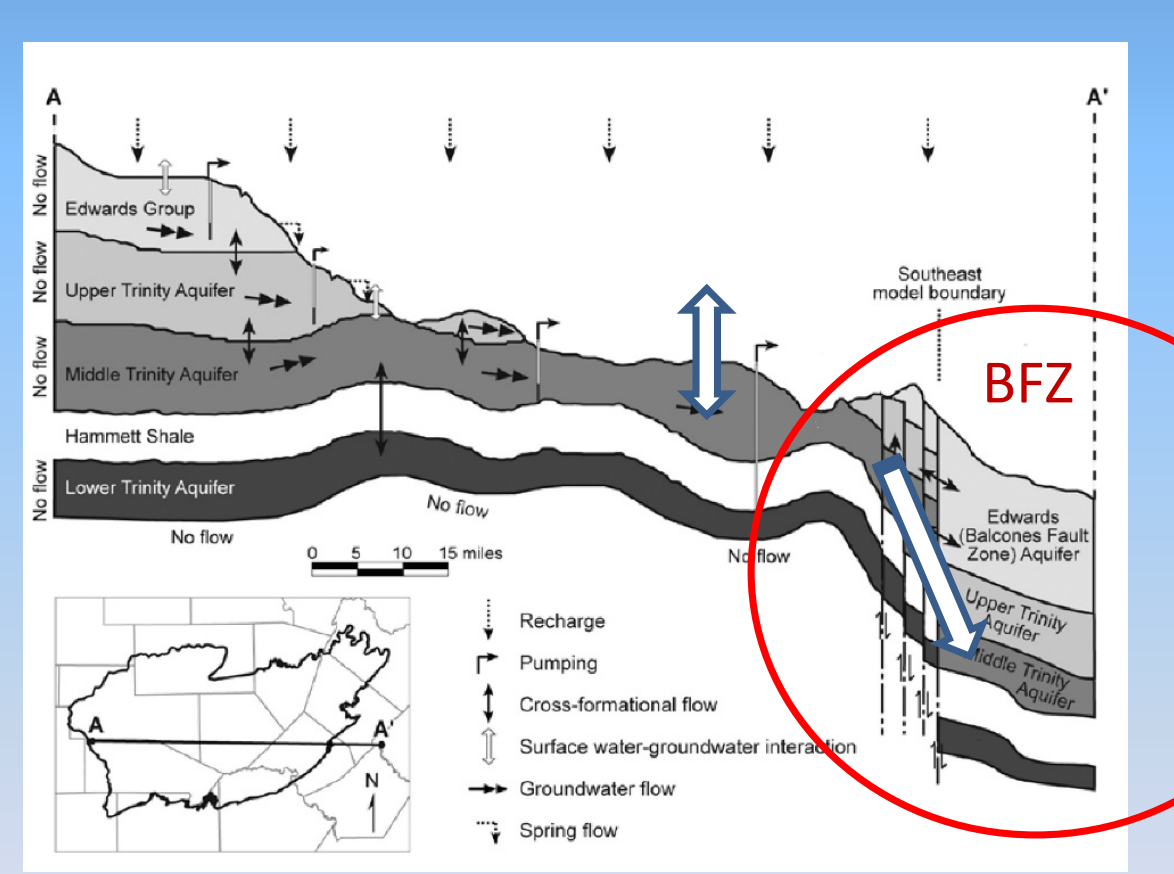

TWDB Report 377, Jones et al., 2011,

- Deeply confined

- Recharge MT is lateral Discharge is unknown

- Fracture and diffuse flow

- Relatively older and variable quality water 


\section{Multiport Monitor Wells}

Edwards and top Upper Trinity are one hydrologic unit.

Edwards Aquifer and Middle Trinity not in hydrologic communication.

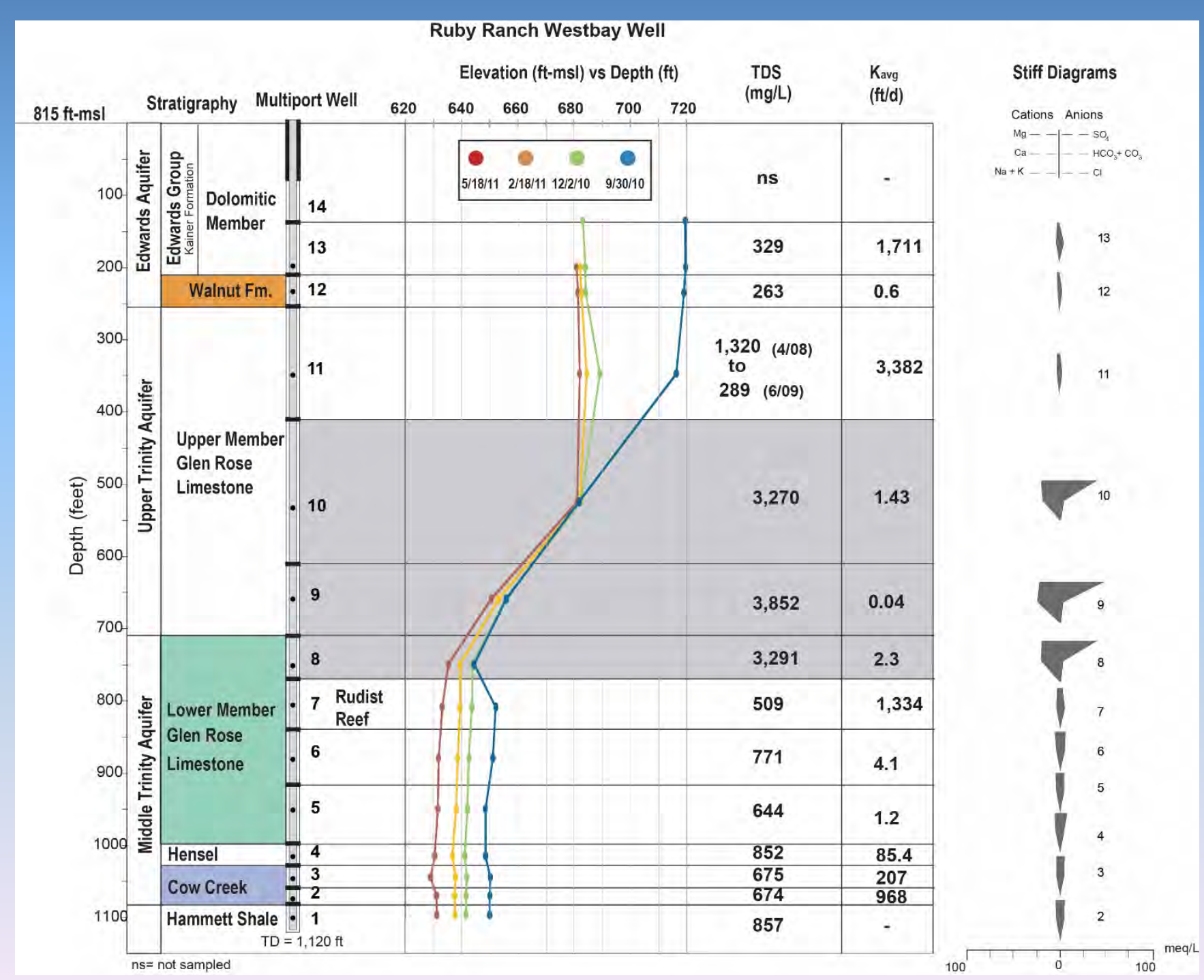

From Hunt et al., 2016 


\section{Interconnection?}

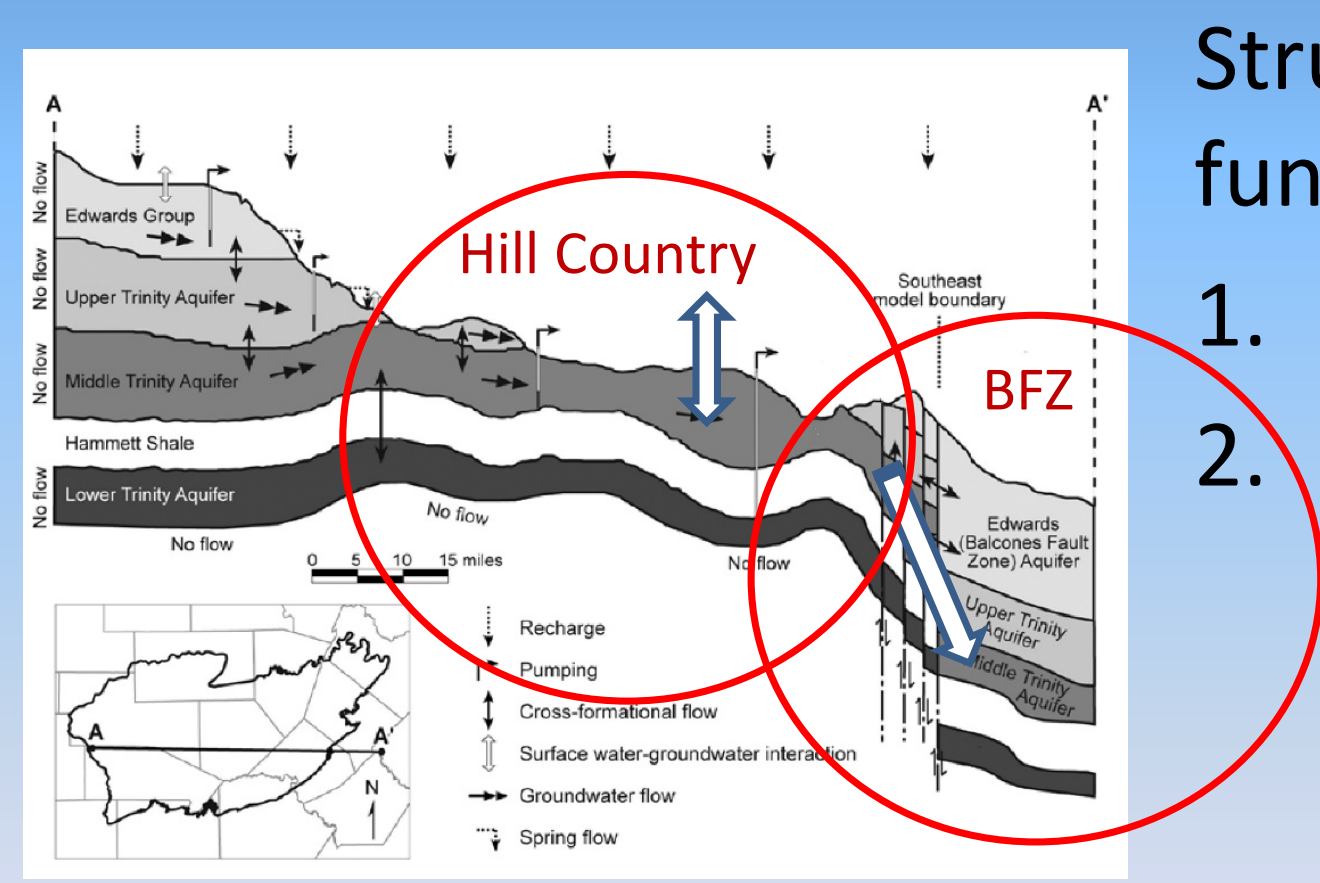

TWDB Report 377, Jones et al., 2011,
Structures perform two functions:

Barriers to flow

Enhance flow or provide mechanism for lateral flow (juxtaposition or relay ramps) 


\section{Relay ramps (transfer zone)}

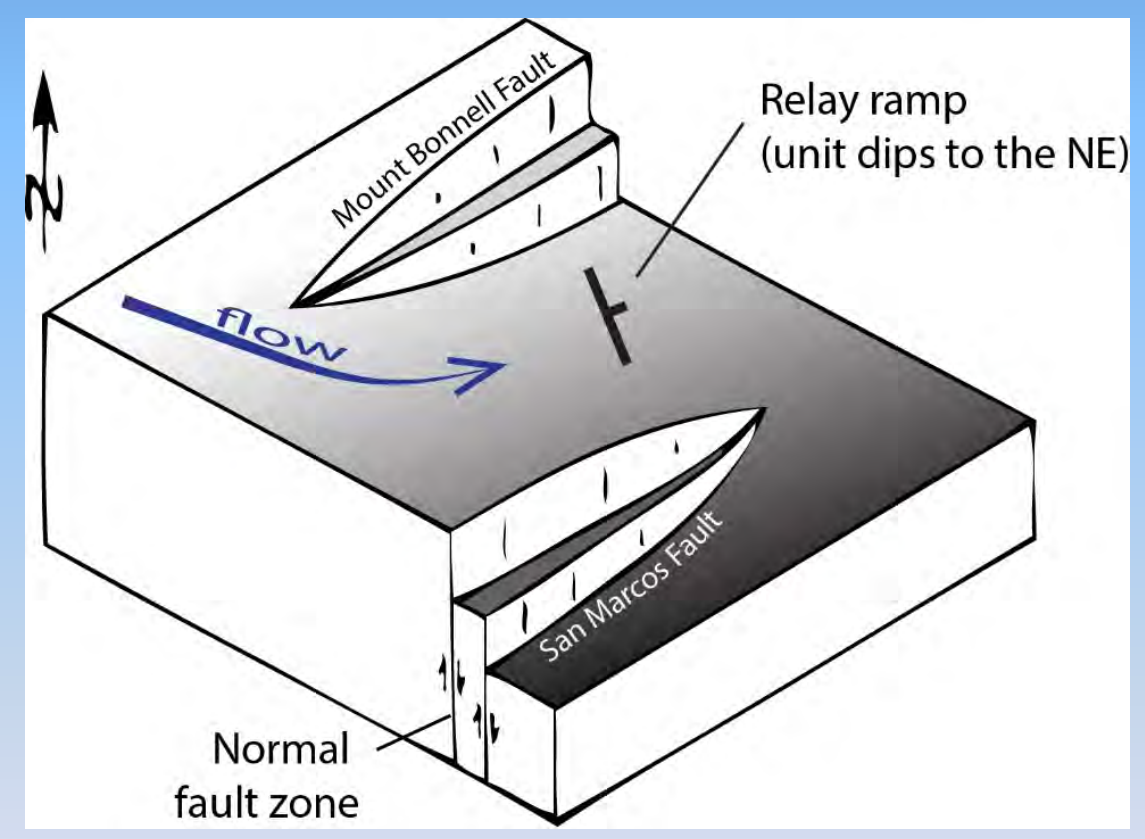

\section{Mechanism for lateral continuity of units within a normal fault zone}

Hunt et al., in review; Modified from Grimshaw and Woodruff, 1986 


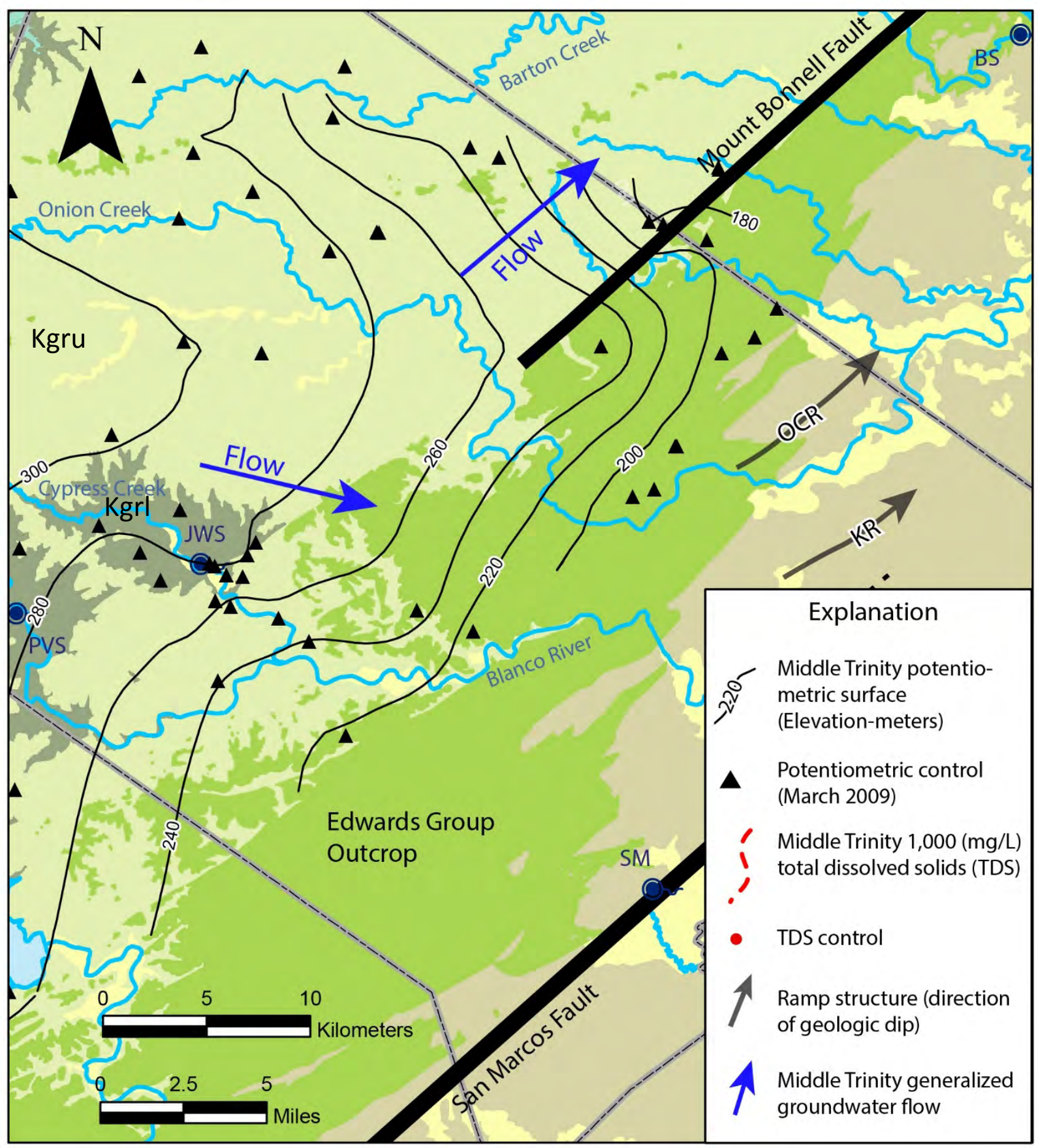

\section{Middle}

Trinity

Aquifer

Flow 


\section{Working Conceptual Model}

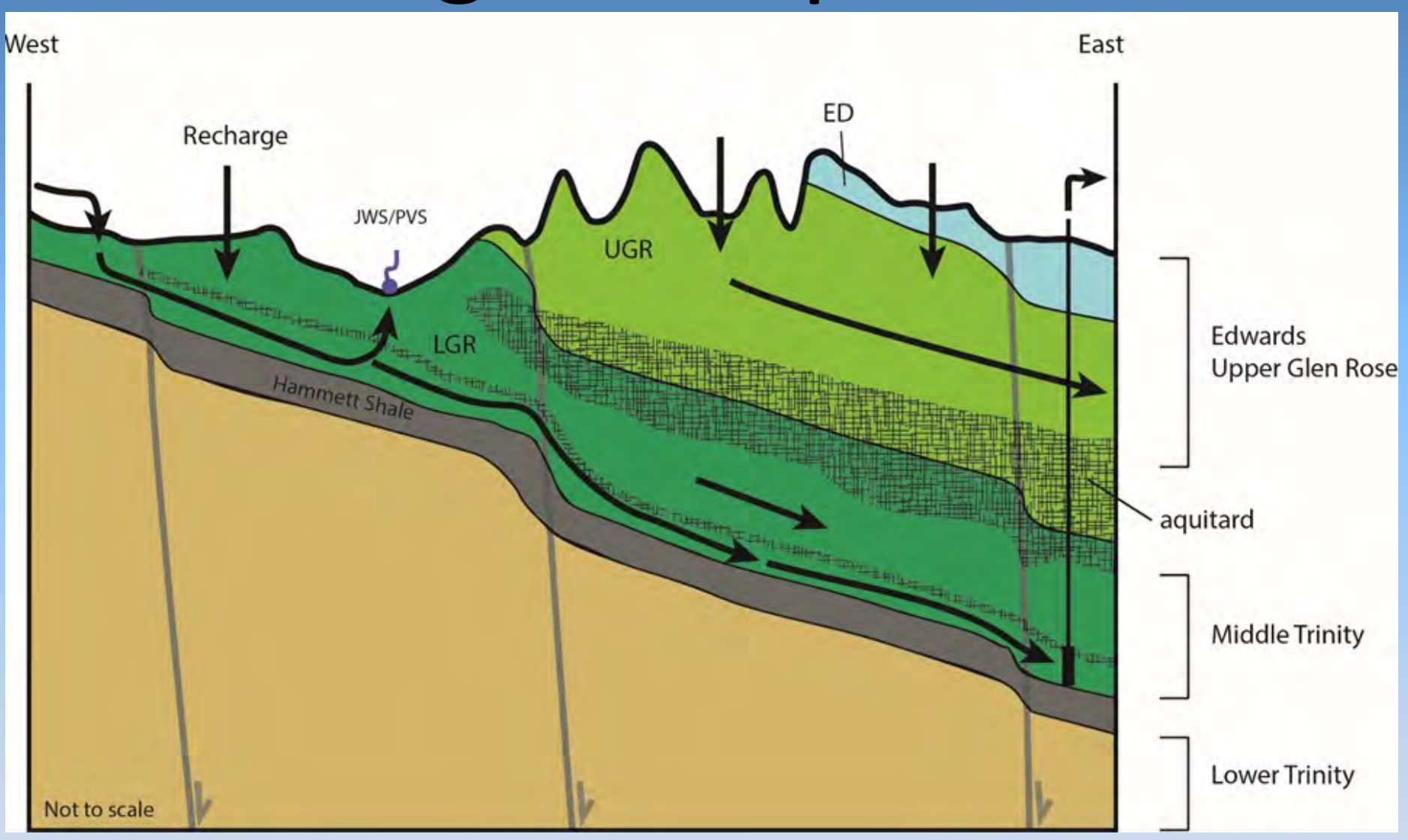

\section{Hill Country Middle Trinity}

- Karstic (caves, springs)

- Surface-groundwater interaction significant

- Conduit to diffuse flow

- Relatively fresh and young water

\section{BFZ Middle Trinity}

- Deeply confined

- Recharge MT is lateral

- Discharge is unknown

- Fracture and diffuse flow

- Relatively older and variable quality water 


\section{Future Work}

Continue to refine and quantify the conceptual model in a collaborative effort through:

- Analyses of recent multiport and hydrogeologic data;

- Other GCD's and entities

- EAA's inter-formational flow study;

- TWDB conceptual model grant (SWRI). 


\section{Thank You!}

Agencies

- TWDB

- EAA

- BSEACD

- HTGCD

- CoA

- TSU

- UT Austin

- Hays County

- Travis County (Vicky Kennedy)

- TPWD (Chad Norris)

\section{Individuals}

- Many land owners, including:

- Halifax Ranch (Bill Johnson)

- Burns Ranch

- Burnett Ranch (Ed Pope)

- Woods Ranch 


\section{Lower Glen Rose}
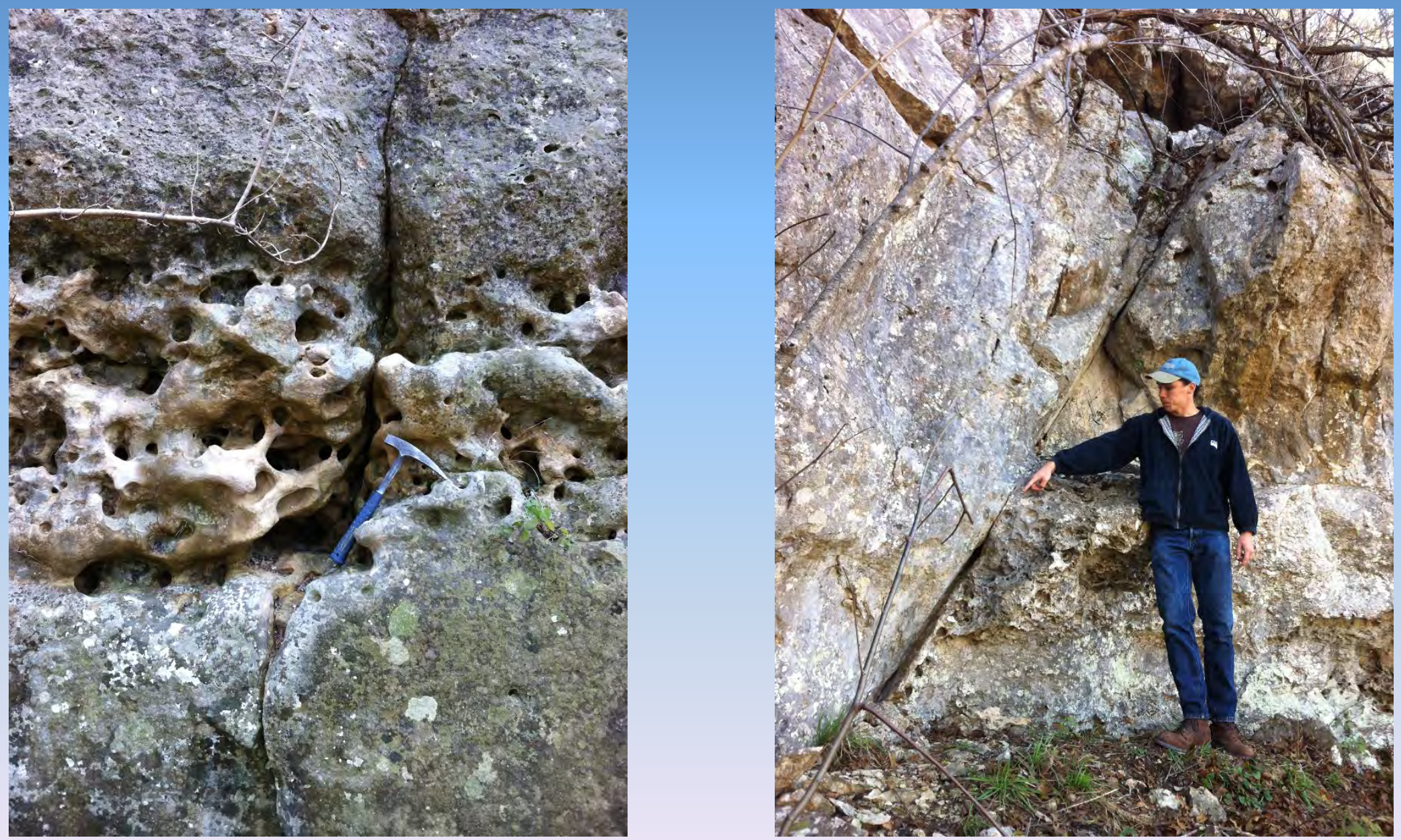